\title{
Distribución y diversidad de hábitats en el humedal de la Reserva Natural Presidente Sarmiento, San Juan, Argentina
}

\section{Distribution and habitats diversity on the wetland of President Sarmiento Natural Reserve, San Juan, Argentina}

\author{
Daniel Germán Flores ${ }^{1 \otimes}$ y Graciela Mabel Suvires ${ }^{1,2}$ \\ ${ }^{1}$ Facultad de Ciencias Exactas, Físicas y Naturales-INGEO-Gabinete de Geología Ambiental. UNSJ. Av. Ignacio de la Roza y Meglioli 5400. Rivadavia, \\ San Juan, Argentina. \\ ${ }^{2}$ CONICET: Consejo Nacional de Investigaciones Cientificas y Técnicas, Argentina. \\ \dflores@unsj-cuim.edu.ar
}

\begin{abstract}
Resumen. En el Área Natural Protegida Presidente Sarmiento (APN), ubicada en la depresión intermontana del valle de Zonda, región del centro-oeste de Argentina, se cartografiaron los factores relieve, consistencia y estado de humedad de los suelos y vegetación, con el fin de contribuir al manejo del humedal. Éste se ubica en la porción distal de un extenso abanico aluvial, en coincidencia con la zona de descarga de agua. Se efectuó un análisis multidisciplinar para establecer relaciones entre la diversidad de hábitats y relieves, la vegetación y los suelos. Se identificaron y clasificaron genéticamente 10 ambientes regionales en la cuenca, donde el ANP ocupa 2 de estos 10 ambientes. A escala local, con fotos aéreas y trabajo de campo, se identificaron 7 sitios con sus variaciones en consistencia de suelos y vegetación. Se clasificaron 37 especies agrupadas en 18 familias. Las Fabaceae y Asteraceae son predominantes. En la reserva, el tamarindo (Tamarix gallica) resultó ser la especie exótica más adaptable; invade y desplaza a las especies nativas. La metodología de trabajo resultó ser muy útil, comenzando desde lo regional hasta el análisis del relieve local.
\end{abstract}

Palabras clave: desiertos argentinos, humedal de descarga de agua subterránea, Tamarix gallica.

\begin{abstract}
In the Natural Area Protected President Sarmiento (APN), situated in an intermountain depression of an arid region of the Zonda valley, a multidisciplinary analysis was performed, to stablish a relationship between habitat diversity, relief, soils and vegetation. This wetland, Provincial Park Reserve President Sarmiento, is lies in a desertic area of central-western part of Argentina. Ten regional environment units within the basin, and 7 sites within the Park were identified and classified. The sites are located in the distal part of an extensive alluvial fan. The survey of the vegetation identified a total of 37 plant species grouped into 18 families. The Fabaceae and Asteraceae are dominants. The tamarindo (Tamarix gallica) was the exotic flora most adaptable to this environment, invading and moving on niches of native species that are particular to the identification ANP. The methology of work followed was very useful beginning from regional to local landforms analysis.
\end{abstract}

Key words: Argentinean deserts, wetland of underwater discharge, Tamarix gallica.

\section{Introducción}

Los humedales tienen funciones que les otorgan un gran valor ambiental atribuido a su alta productividad biológica y diversidad de hábitats, y a su participación en la estabilización de procesos hidrológicos y como filtros naturales (Brown y Lant, 1999; Bodini et al., 2000). Son de gran importancia tanto para la conservación de la diversidad como para el desarrollo de las comunidades humanas asociadas a ellos (Gonzales, 1993). En las últimas décadas, los humedales se han revalorizado, especialmente

Recibido: 11 mayo 2010; aceptado: 03 octubre 2011 como núcleos de biodiversidad y reguladores hidrológicos, debido a que estos ecosistemas no sólo contribuyen al sostenimiento de los circuitos migratorios de aves (Almazán y Navarro, 2006; Flores y Suvires, 2009) sino además a la diversidad de especies de plantas nativas (De La Barrera, 2008; Flores, 2008). Las condiciones ambientales de los terrenos de los humedales propician el uso de la tierra, que conjuntamente con la modificación del régimen hídrico y la introducción de especies exóticas alteran la dinámica de estos sistemas, comprometiendo su flora y fauna original (Brinson y Malvárez, 2002). En estos ecosistemas cada factor ambiental tiene distintos efectos sobre los demás (De La Barrera, 2008); el estudio de las causas de la existencia y de la dinámica de los humedales ofrece resultados 
que señalan la importancia del conocimiento de las situaciones locales en estos hábitats.

Este trabajo tiene como objetivo analizar los factores ambientales, el relieve, la vegetación y la consistencia y estado de humedad de los suelos del Área Natural Protegida Parque Provincial Presidente Sarmiento (APN), ubicado en el centro-oeste árido de Argentina, con el fin de contribuir al manejo del humedal. Se utiliza una metodología sencilla de trabajo, desde el enfoque regional de la cuenca, con la identificación de 10 ambientes de relieves regionales, hasta alcanzar un nivel de estudio detallado, con la identificación de 7 sitios de relieves y sus correspondencias en suelos y vegetación.

En Argentina, el territorio de la provincia de San Juan, posee algunas áreas de protección nacional e internacional, correspondiendo 720 ha al Área Natural Protegida en estudio. En 2005, por la Ley Provincial 7586, fue declarada reserva de usos múltiples y Parque Provincial Presidente Sarmiento. La misma, posee cuerpos de agua dulce, permanentes y temporarios, de contornos dinámicos y abundante vegetación emergente (Flores, 2008). El valle de Zonda muestra un territorio de características heterogéneas que comprende complejas interacciones entre la configuración espacial y los procesos ecológicos. En esta región central del Monte, la heterogeneidad espacial de la vegetación y de sus condiciones ambientales son similares a las de otras regiones áridas y semiáridas del mundo (Suvires, 2000). Las observaciones detalladas, principalmente de campo, estuvieron dirigidas a las variaciones en los relieves o micro-formas; se efectuaron con apoyo de fotogramas y fotografías aéreas, lo que permitió establecer relaciones entre las unidades territoriales o hábitats, las propiedades de los suelos y las especies vegetales (nativas-exóticas) presentes, y proveer nuevos datos e información para la comprensión de este ecosistema dentro del contexto regional de la cuenca de Zonda.

\section{Materiales y métodos}

Área de estudio. En la depresión tectónica del valle de Zonda, provincia de San Juan, centro-oeste de Argentina (31 ${ }^{\circ} 33^{\prime}$ S, $68^{\circ} 42^{\prime}$ O) se ubica el humedal del Área Natural Protegida, Reserva de Usos Múltiples y Parque Provincial Presidente Sarmiento, con una superficie de 720 ha, a una altura promedio de $760 \mathrm{~m} \mathrm{snm}$ (Fig. 1).

El área de estudio se desarrolla en la parte distal de un extenso abanico aluvial del río San Juan, limitada hacia el este por la sierra de Marquesado. Desde el punto de vista geológico-geomorfológico, este valle es una depresión tectónica e intermontana, longitudinal, que se extiende entre 2 alineamientos montañosos morfoestructurales, de disposición norte-sur, pertenecientes a precordillera central (sierra
Alta de Zonda-Cerro Blanco) al oeste, y a la precordillera oriental (sierra Marquesado-Sierra Chica de Zonda) hacia el este (Ortiz y Zambrano, 1981).

La zona de estudio está incluida en la Provincia Fitogeográfica del Monte (Cabrera, 1994; Bertiller et al., 2009). La precipitación media anual es de $86.1 \mathrm{~mm}$ y la temperatura media de $25.7^{\circ} \mathrm{C}$. El clima es seco-desértico. Las descargas de agua que recibe el humedal provienen desde el oeste de los caudales estacionales del río San Juan, el que se alimenta, en la Cordillera de los Andes, de precipitaciones níveas y derretimiento de glaciares, y desde el norte del embalse artificial de Ullum.

Trabajo de laboratorio/gabinete. Mediante fotointerpretación a escala 1:20 000 y fotogramas a escala 1:10 000, con base en diferencias entre las distintas tonalidades, diseños y texturas de las imágenes fotográficas, se identificaron, primero, 10 unidades regionales (Fig. 2) y luego, 7 diferentes sitios (Fig. 3). Las fotografías aéreas fueron provistas por el Centro de Fotogrametría y Catastro de San Juan (vuelo IGM 1994). De manera simultánea a la fotoidentificación y fotointerpretación de las unidades territoriales se elaboraron referencias descriptivas y de clasificación geomorfológica preliminar y previa al apoyo de campo. En gabinete se identificaron y clasificaron las especies vegetales que no pudieron clasificarse en el campo, utilizando catálogos y manuales de vegetación de monte.

Trabajo de campo. En los distintos sitios delimitados con fotointerpretación se efectuaron recorridos de campo a pie, registrando las variaciones de los perfiles de suelos en aquellos lugares accesibles y sin agua en superficie; se abrieron pozos de $35 \mathrm{~cm}$ de profundidad y se determinó textura manual y consistencia al tacto (en seco, húmedo y mojado) en cada uno de los cambios de relieves o sitios,

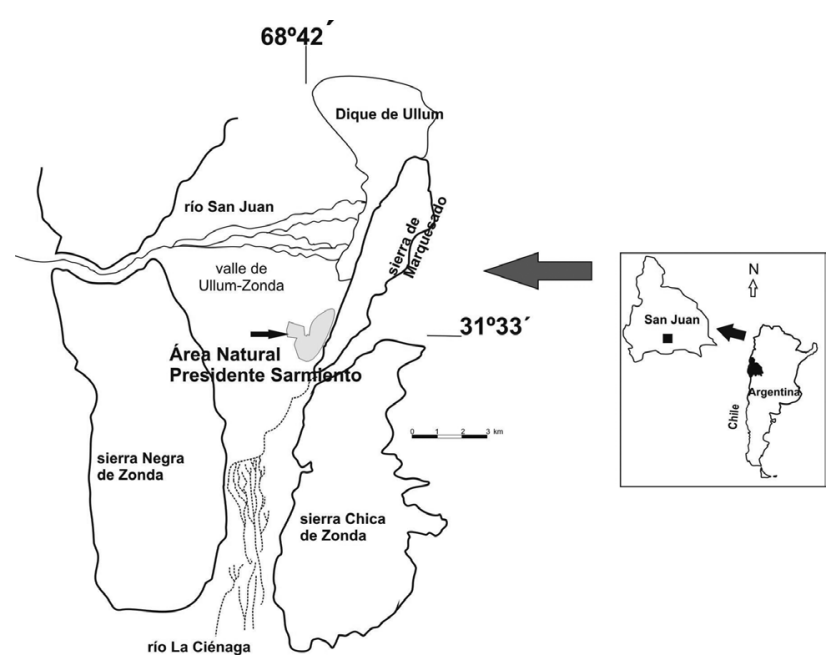

Figura 1. Ubicación del área de estudio. Valle de Zonda, San Juan, Argentina. 


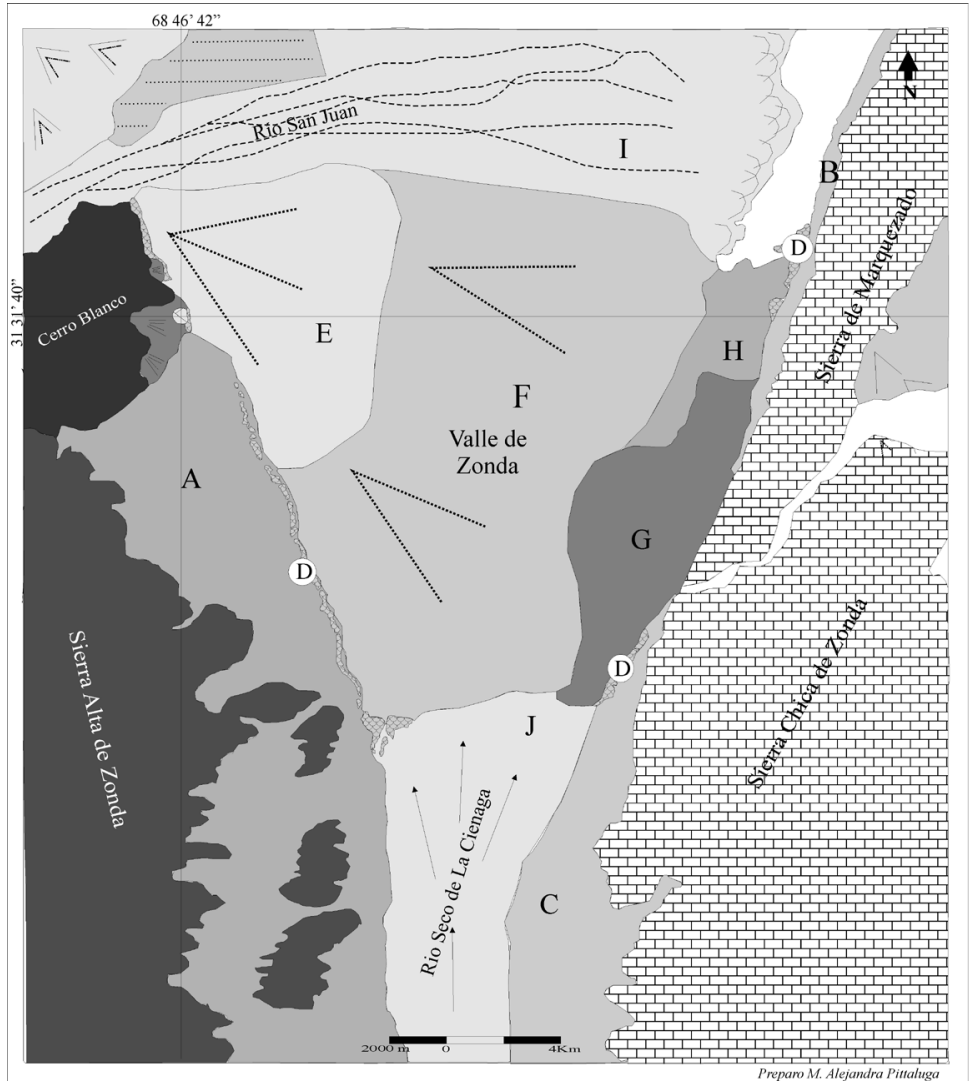

Unidades Geomorfológicas

\begin{tabular}{|c|c|}
\hline A & Piedemonte Sierra Alta de Zonda \\
\hline B & Piedemonte Sierra de Marquesado \\
\hline $\mathrm{C}$ & Piedemonte Sierra Chica de Zonda \\
\hline D & Depósitos Lacustres holocenos \\
\hline $\mathbf{E}$ & Sector Proximal del abanico aluvial \\
\hline $\mathbf{F}$ & Sector Medio del abanico aluvial \\
\hline G & Sector Distal del abanico con suelos húmedos. \\
\hline H & Sector Distal del abanico con agua en superficie \\
\hline I & Cauce Actual y Llanura de inundación del río San Juan \\
\hline $\mathbf{J}$ & Cauce actual-llanura de inundación del río Seco de la Ciénaga \\
\hline
\end{tabular}

G y H ambientes que ocupa la Reserva Natural del Parque Presidente Sarmiento

Abanico aluvial

$\longrightarrow$ Dirección de escurrimiento

Calizas cambro -ordovícicas

Areniscas y pelitas del Devónico

Andesitas y Dacitas del Mioceno

Figura 2. Unidades geomorfológicas regionales en el Valle de Zonda.

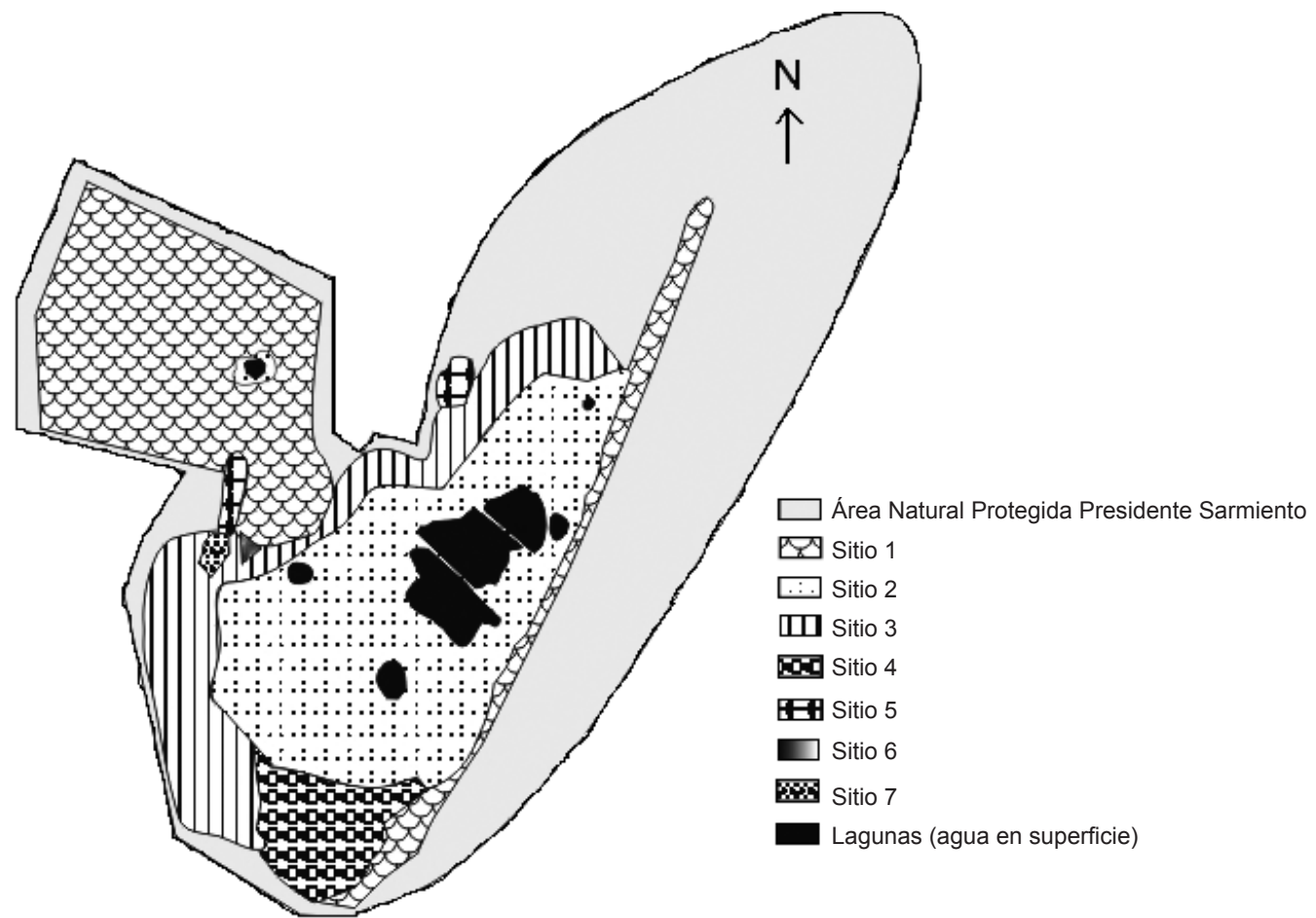

Figura 3. Zonificación de unidades de tierra, a escala aprox. 1:25 000 en detalle (sitios) del ANP. 
Cuadro 1. Relaciones entre relieves-vegetación-consistencia del suelo de los sitios del Área Natural Protegida Presidente Sarmiento. Especies adicionales: S1: Fabaceae: Geoffroea decorticans (Guillies ex Hook et Arn.) Bukart. S3: Poaceae: Muhlenbergia asperifolia (Nees et Meyen ex Trin.) Parodi.; Phragmites australis Cav. (Trin. ex Steud). Asteraceae: Wedelia glauca (Ortega) Hoffmann ex Hicken.; Baccharis salicifolia (Ruiz et Pavón) Pers.; Senecio subulatus D. Don ex Hook et Arn

\begin{tabular}{|c|c|c|c|c|c|c|}
\hline \multirow{2}{*}{$\begin{array}{l}\text { Unidad } \\
\text { regional }\end{array}$} & \multirow{2}{*}{$\begin{array}{c}\text { Sitio } \\
\text { paisaje }\end{array}$} & \multirow{2}{*}{$\begin{array}{l}\text { Ubicación en } \\
\qquad A N P\end{array}$} & \multirow[b]{2}{*}{ Relieve } & \multirow[b]{2}{*}{ Suelo } & \multicolumn{2}{|c|}{ Vegetación dominante } \\
\hline & & & & & Familia & Especie \\
\hline \multirow[t]{19}{*}{$\begin{array}{l}\text { Sector distal } \\
\text { del abanico con } \\
\text { suelos húmedos } \\
\text { (G) }\end{array}$} & $\mathrm{S} 1$ & $\begin{array}{l}\text { Oeste del } \\
\text { ANP, en la } \\
\text { porción distal } \\
\text { del abanico }\end{array}$ & $\begin{array}{l}\text { Plano a } \\
\text { plano - } \\
\text { inclinado }\end{array}$ & $\begin{array}{l}\text { Texturas finas } \\
\text { con algunos } \\
\text { rodados } \\
\text { pequeños de } \\
\text { caliza y gravillas. } \\
\text { Consistencia: }\end{array}$ & Fabaceae & $\begin{array}{l}\text { Prosopis strombulifera Lam. } \\
\text { Prosopis chilensis Stunz. } \\
\text { Prosopis flexuosa DC. } \\
\text { Acacia aroma Guillies. } \\
\text { Medicago sativa L. } \\
\text { Melilotus albus Desr. }\end{array}$ \\
\hline & & & & $\begin{array}{l}\text { ligeramente } \\
\text { duro, no plástico }\end{array}$ & Chenopodiaceae & $\begin{array}{l}\text { Atriplex deserticola } \text { Phil, } \\
\text { Atriplex crenatifolia Chodat. }\end{array}$ \\
\hline & & & & y ligeramente & Zygophyllaceae & Larrea divaricata $\mathrm{Cav}$ \\
\hline & & & & adhesivo & Solanaceae & $\begin{array}{l}\text { Solanum eleagnifolium Cav. } \\
\text { Lycium tenispinosum Miers. } \\
\text { Grabowskia obtusa Am. }\end{array}$ \\
\hline & $\mathrm{S} 2$ & Rodea al & Suavemente & Mojado en la & Typhaceae & Typha domingensis Pers. \\
\hline & & núcleo & deprimido & mayor parte & Juncaceae & Juncus acutus L. \\
\hline & & de agua. & cóncavo & del año. Tiene & Equisetaceae & Equisetum giganteum $\mathrm{L}$. \\
\hline & & $\begin{array}{l}\text { De límite } \\
\text { indefinido }\end{array}$ & & $\begin{array}{l}\text { plasticidad y } \\
\text { adhesividad } \\
\text { moderada }\end{array}$ & Umbelíferas & Hydrocotyle bonariensis Lam. \\
\hline & $\mathrm{S} 3$ & $\begin{array}{l}\text { Transición } \\
\text { se ubica }\end{array}$ & $\begin{array}{l}\text { Plano a } \\
\text { plano- }\end{array}$ & $\begin{array}{l}\text { Estos suelos } \\
\text { tienen mayor }\end{array}$ & Poaceae & $\begin{array}{l}\text { Paspalum dilatatum Poir. } \\
\text { Cynodon dactylon L. }\end{array}$ \\
\hline & & bordeando al & inclinado & humedad que & Salicáceas & Salix humboldtiana Willd. \\
\hline & & $\mathrm{S} 2 \mathrm{y}$ en partes & & los del S1 pero & Solanaceae & Solanum eleagnifolium Cav. \\
\hline & & próximo al S1 & & $\begin{array}{l}\text { menos que los } \\
\text { del S2 }\end{array}$ & Asteraceae & $\begin{array}{l}\text { Tessaria absinthioides DC. } \\
\text { Solidago chilensis Meyen. }\end{array}$ \\
\hline & & & & & Malvaceae & Spharealcea miniata Cav. \\
\hline & & & & & Fabaceae & $\begin{array}{l}\text { Prosopis strombulifera Lam. } \\
\text { Prosopis flexuosa DC. } \\
\text { Medicago sativa L. }\end{array}$ \\
\hline & & & & & Ranunculáceas & $\begin{array}{l}\text { Clematis montevidensis } \\
\text { Spreng. }\end{array}$ \\
\hline & S4 & Sector sur & Plano & $\begin{array}{l}\text { Consistencia del } \\
\text { suelo es blanda }\end{array}$ & Tamaricaeae & Tamarix gallica $\mathrm{L}$. \\
\hline & S5 & Sector norte & Plano & $\begin{array}{l}\text { Consistencia del } \\
\text { suelo es blanda } \\
\text { (en seco) }\end{array}$ & Salicaceae & Populus nigra $\mathrm{L}$. \\
\hline & S6 & Sector oeste & Plano & $\begin{array}{l}\text { Consistencia } \\
\text { ligeramente dura } \\
(\text { en seco) }\end{array}$ & Casuarinaceae & $\begin{array}{l}\text { Casuarina cunninghamiana } \\
\text { Miq. }\end{array}$ \\
\hline & S7 & Sector oeste & Plano & $\begin{array}{l}\text { Consistencia } \\
\text { ligeramente dura } \\
\text { (en seco) }\end{array}$ & Myrtaceae & Eucalyptus tereticornis $\mathrm{Sm}$. \\
\hline
\end{tabular}

siguiendo el método de Etcheveherre (1976). Entre las propiedades físicas de los suelos, la consistencia es una característica fundamental en los ambientes de humedales que permite analizar la variación del contenido de humedad en los suelos y su comportamiento físico-mecánico en relación con los aportes de agua subterránea o superficial. La consistencia se encuentra vinculada a la textura y a la estructura, principalmente, es la coherencia entre las partículas del suelo y varía según el estado de humedad, por lo que conviene determinarla en seco, húmedo y mojado.

Durante la primavera del 2009, se realizó una evaluación de las especies de plantas en cada uno de los sitios mediante 2 transectos de $30 \mathrm{~m}$ de longitud con 4 parcelas de $6 \mathrm{~m}^{2}$, separadas $2 \mathrm{~m}$ entre ellas. Se registró presencia- 
ausencia de especies de plantas nativas y exóticas. El estudio se realizó teniendo en cuenta la sectorización territorial antes señalada.

\section{Resultados}

Las figuras. 2 y 3 muestran la distribución de los hábitats regionales y locales que existen tanto en la cuenca de Zonda como en el Área Natural Protegida (ANP), respectivamente. En la figura 3 se identificaron 7 sitios, todos ubicados en la parte distal del antiguo y abandonado abanico aluvial del río San Juan (Fig. 2, unidades G y H).

Con métodos geofísicos y geoeléctricos, Rocca, (1969) estudió la hidrogeología de la cuenca de Zonda, definiendo el abanico aluvial depositado por el río San Juan como una gran unidad geomorfológica regional antigua. Este abanico se habría formado en la depresión de Zonda en el Pleistoceno tardío, debido a los grandes aportes hídricos y descargas de sedimentos aluvionales provenientes de los derretimientos y retrocesos de los glaciares de los Andes centrales (Colombo et al., 2000; Lloret y Suvires, 2006). La depresión tectónica de Zonda está asociada a los bordes de las áreas montañosas de Precordillera. En general, estas cuencas son áreas deprimidas, rellenas con materiales sedimentarios fluviales y aluviales de espesores potentes que pueden sobrepasar los $900 \mathrm{~m}$, como en Zonda (Zambrano, 1985). Estos relieves son el resultado de una acción combinada de una actividad tectónica que generó una depresión primigenia de una intensa depositación aluvional posglaciaria.

El abanico aluvial del río San Juan, en Zonda, se desarrolla con una pendiente moderada $(<5 \%)$, y presenta su ápice a la altura del cerro Blanco, y alcanza una longitud media de $23 \mathrm{~km}$, con un largo y un ancho de aproximadamente $20 \mathrm{~km}$, medidos en su parte distal (Suvires, 2008). Dentro de este relieve se diferenciaron 3 sectores: uno proximal (unidad E), otro medio (unidad F) y otro más, distal, al pie del abanico. En éste último se reconocen 2 sitios; uno, donde el agua aflora en superficie (unidad G) y otro donde el agua está en el subsuelo y temporalmente próxima a la superficie (unidad $\mathrm{H}$ ). Ambos sitios, F y H, corresponden al área del Parque Presidente Sarmiento. El cauce del río San Juan (unidad I) es el sistema fluvial actual y constituye una unidad representada tanto por el cauce como por la llanura de inundación. El cauce del río San Juan está obstaculizado en su recorrido por el embalse de Ullum, utilizado para riego aguas abajo en el valle de Tulum.

La planicie aluvial y cauce del río Seco de la Ciénaga (Unidad J) constituye un relieve de menor tamaño pero no de menor importancia, dado que contribuye al valle de Zonda con aportes de sedimentos y agua, principalmente de aluviones estivales torrenciales de alta peligrosidad y muy erosivos.

Dentro del ANP y en los alrededores se delimitaron los 7 sitios (Fig. 3), investigados en detalle. El estudio de campo de la vegetación de los sitios, permitió clasificar y determinar un total de 37 especies vegetales, agrupadas en 19 familias. Las muestras de suelos analizadas presentaron variaciones en la consistencia, en los estados seco, húmedo y mojado, desde friable, adhesivo, blando, hasta ligeramente duro (Cuadro 1).

\section{Discusión}

Varios son los métodos utilizados para intentar conocer la distribución de hábitats en las regiones desérticas del mundo, en particular, la dinámica de los mismos para implementar planes de manejo o de conservación de ambientes como los humedales. Pero, los autores de este trabajo, al provenir de diferentes especialidades, han considerado los paisajes o relieves como unidades ambientales que fusionan una serie de factores y de procesos, susceptibles de medirse a través de indicadores, como la posición o ubicación del sitio en relación con la cuenca; las características y propiedades de sus suelos y depósitos geológicos; el tipo de micro-relieve ( plano, cóncavo, convexo); el tipo y densidad de la vegetación, y -aunque en este trabajo no se contempló por no ser el objetivo- hasta el tipo de actividades antrópicas presentes. En total acuerdo con Sayago, (1982) y Pittaluga y Suvires, (2009), la cartografía observada en detalle (paisajes-sitios-micrositios) es la más adecuada, dado que cada uno de los sitios tiene una vinculación con el tipo y densidad de vegetación, consistencia y grado de humedad del suelo, y hasta con la capacidad de uso de cada uno de ellos. En el humedal y alrededores se observó que ante la dinámica hidrológica de la cuenca, proveniente de la variabilidad periódica de los caudales del río San Juan y de los niveles del embalse de Ullum, el recurso natural más vulnerable es la vegetación asociada a las condiciones de humedad de los suelos, especialmente la relación vegetación nativa vs. exótica. El humedal del ANP, Parque Presidente Sarmiento, está inserto en una región extremadamente árida y sin lluvias, alimentado por descargas subterráneas vinculadas al cauce del río San Juan. Flores y Suvires, (2009) lo han tipificado como humedal de descarga de agua subterránea con una alta vulnerabilidad a los impactos antrópicos de la cuenca y a los cambios (interanuales o interperiódicos) de los caudales del río San Juan. El avance de Tamarix gallica sobre las especies nativas del lugar impacta significativamente debido a su alto consumo de agua y evapotranspiración. Además, Vitousek et al. (1996) señalan que algunas especies exóticas cambian las condiciones edáficas originales, 
al aumentar la salinización del suelo. Según Anderson et al. (1993), la detección temprana, la cartografía a tiempo y el monitoreo frecuente de las distribuciones de plantas invasoras son fundamentales para la gestión y conservación los recursos naturales. En este trabajo se rescata y se pone en evidencia un método de trabajo cartográfico desde lo regional (Fig. 2) hasta el detalle (Fig.3), poniendo de manifiesto que la distribución de los hábitats a escala de sitio debe contemplar una primera visión regional en función de la cuenca. No se podrá entender cabalmente la dinámica y comportamiento local de los sitios, con sus distintas características (relieve, suelos, vegetación, otros), si éstos no se vinculan con el ecosistema regional o unidades de la cuenca. La delimitación de los 7 sitios con sus características, expresadas en el Cuadro 1, son resultados para considerarse en los lineamientos de planes de manejo del ANP. Los sitios S5 al S7 presentan una vegetación asociada a una especie única; 2 de éstos, S5 y S7, están influenciados por la reforestación, mientras que S4 y S6, que coinciden con los sitios más vulnerables a la dinámica hidrológica del humedal, contienen una sola especie de tipo exótico. En este trabajo se ha observado que, si bien, los sitios expresan o delimitan relieves detallados diferentes, éstos son dinámicos temporalmente $\mathrm{y}$, en consecuencia, modifican su dinámica vegetacional. Es evidente que el avance de algunas especies exóticas, como tamarindo y casuarina, producirá un retardo en la recuperación de las condiciones ambientales primigenias del ecosistema. Se concluye que para el manejo del área del humedal ANP Parque Presidente Sarmiento, la metodología de trabajo utilizada es recomendable, sugiriendo siempre mantener una estrecha correlación escalar entre la cartografía de los relieves y la de la vegetación, como por ejemplo el nivel de sitio se corresponde con el de especie.

\section{Literatura citada}

Almazán-Núñez, R. y A. Navarro. 2006. Avifauna de la subcuenca del río San Juan, Guerrero, México. Revista Mexicana de Biodiversidad 77:103-114.

Anderson, G. L., J. H. Everitt, A. J. Richardson y D. E. Escobar. 1993. Using satellite data to map false broomweed (Ericameria austrotexana) infestations on south Texas rangelands. Weed Science 7:865-871.

Bertiller, M. B., L. Marone, R. Baldi y J. Ares. 2009. Biological interactions at different spatial scales in the Monte desert of Argentina Journal of Arid Environments 73:212-221.

Bodini, A., A. Ricci y P. Viaroli. 2000. A multimethodological approach for the sustentable management for perifluvial wetlands of the Po river (Italy). Environmental Management 26:59-72.

Brinson, M. M. y I. Malvarez. 2002. Temperate freshwater wetlands: types, status, and threats. Environmental Conservation 29:115-133.

Brown, P. y C. Lant. 1999. The effect of wetland mitigation banking on the achievement of no-net-loss. Environmental Management 23:333-345.

Cabrera, A. L. 1994. Regiones fitogeográficas argentinas. In Enciclopedia argentina de agricultura y jardinería, $t$ 2, W.F. Kugler (ed.). ACME, Buenos Aires. 57 p.

Colombo, F., P. Busquets, E. Ramos, J. Vergés y D. Ragona. 2000. Quaternary alluvial terraces in an active tectonic region: the San Juan River Valley, Andean Ranges, San Juan Province, Argentina. Journal of South American Earth Sciences 13:611-626.

De La Barrera, E. 2008. Recent invasion of buffel grass (Cenchrus ciliaris) of a natural protected area from the southern Sonoran Desert. Revista Mexicana de Biodiversidad 79:385-392.

Etcheveherre, P. 1976. Normas de reconocimientos de suelos. Instituto Nacional de Tecnología Agropecuaria (INTA)Castelar, Buenos Aires. 115 p.

Flores, D. 2008. Caracterización biológica del valle de Zonda. In Zonda: un oasis para soñar. Instituto y Museo Manzini, Zonda, Archivo provincia San Juan, Gobierno de San Juan. p. $37-48$.

Flores, D. y G. Suvires. 2009. Susceptibilidad del área natural protegida (ANP) y Parque Provincial Presidente Sarmiento, en la provincia de San Juan. Encuentro Internacional E-ICES, 5 de noviembre de 2009, Malargüe, Mendoza. p. 105-112.

Gonzales, A. 1993. Bases para la conservación de los humedales de la costa de Toltén (IX Región). Informe proyecto DIUCT $N^{\circ}$ 99-4- 04. Dirección de Investigación, Universidad Católica de Temuco, Araucania. p.1-56.

Lloret, G. y G. Suvires, 2006. Ground water basin of the Tulum Valley, San Juan, Argentina: a morphohydrogeologic analysis of its central area. Journal of South American Earth Sciences 21:267-275.

Ortiz, A. y J. Zambrano. 1981. La provincia geológica precordillera Oriental. VIII Congreso Geológico Argentino, 20-26 setiembre, 1981, San Luis, Actas III. Asociación Geológica Argentina, Buenos Aires. p. 34-42.

Pittaluga, M. A. y G. M. Suvires. 2009. Geomorfología dinámica en depósitos cuaternarios del valle de Zonda para el ordenamiento territorial. Provincia de San Juan. In Actas, Encuentro Internaional E-ICES, 5 de noviembre de 2009, Malargüe, Mendoza. p. 231-235.

Rocca, J. A. 1969. Geología de los valles de Tulum y UllumZonda. Provincia de San Juan, T 1 y 2. PASNOA. P: 1-210, Tomos 1 y 2. Centro Regional de Aguas Subterráneas, San Juan.

Sayago, J. M. 1982. Las unidades de relieve como estudio integrado de los recursos naturales. Revista Acta Lilloana. 
Instituto INGEMA, Universidad de Tucumán, San Miguel. p. 1-18.

Suvires, G. 2000. Mapa geomorfológico de la provincia de San Juan, escala 1:1.000.000. In Atlas de cartografías temáticas de las provincias andinas argentinas, tomo II. E.M. Abraham y F. R. Martínez (eds.). CRICYT-IADIZA-Junta Andalucía, Mendoza.

Suvires, G. M. 2008. Geomorfología del valle Ullum, Zonda. In Zonda: un oasis para soñar. Instituto y Museo Manzini,
Zonda. Registro del Archivo de la provincia de San Juan. Congreso Argentino de Archivo, 2008, San Juan, tomo 1, p.37-50.

Vitousek, P., C. M. D'Antonio, L. Loope y R. Westbrooks. 1996. Biological invasions as global environmental change. American Scientist 84:468-478.

Zambrano, J. J. 1985. Actualización geológica del valle de Zonda. Centro Regional de Aguas Subterráneas-Instituto Nacional de Aguas (CRAS-INA), San Juan. p. 1-120. 